\title{
A Case of Temporal Bone Abscess in an Immunocompromised Patient
}

\author{
Andrew Tan Kie Kion, Nik Adilah Nik Othman
}

Otorhinolaryngology-Head \& Neck Surgery, School of Medical Sciences, Health Campus, Universiti Sains Malaysia

\begin{abstract}
Introduction: Acute otitis media (AOM) is an otological disease that is usually self-limiting. However, certain AOM complicates with abscess development over the deep neck cavity (Bezold's abscess), occiptal abscess (Citelli's) or beneath the temporal muscle (Luc's abscess). Luc's abscess was described as a more benign form of AOM that seldom require extensive surgical drainage.
\end{abstract}

Case report: We report a case of a subperiosteal temporal bone abscess in an immunocompromised patient, which resolved with local drainage of abscess and cultureoriented antimicrobial.

Conclusion: Luc's abscess is a rare form of the complication of otitis media, associated with relatively lower morbidity, better prognosis, and requires a limited surgical intervention. High resolution computed tomography (HRCT) temporal bone is highly recommended to evaluate the extent of the disease and to decide for further treatment strategy. The choice of surgery should also be tailored to the patient's premorbid factor.

Keywords: Luc's abscess, subperiosteal temporal bone abscess, immunocompromised, acute otitis media, complications 